\title{
On periodic solutions of a discrete Nicholson's dual system with density-dependent mortality and harvesting terms
}

\author{
Rajendiran Eswari ${ }^{1}$, Jehad Alzabut ${ }^{2,33^{*}}$ (D), Mohammad Esmael Sameji, ${ }^{4 *^{*}}$ (D) and Hui Zhou ${ }^{6}$
}

${ }^{*}$ Correspondence:

jalzabut@psu.edu.sa;

mesamei@basu.ac.ir;

mesamei@gmail.com

${ }^{2}$ Department of Mathematics and

General Sciences, Prince Sultan

University, Riyadh, Saudi Arabia

${ }^{4}$ Department of Mathematics, Bu-Ali

Sina University, Hamedan, Iran

Full list of author information is

available at the end of the article

\section{Springer}

\begin{abstract}
In this study, we discuss the existence of positive periodic solutions of a class of discrete density-dependent mortal Nicholson's dual system with harvesting terms. By means of the continuation coincidence degree theorem, a set of sufficient conditions, which ensure that there exists at least one positive periodic solution, are established. A numerical example with graphical simulation of the model is provided to examine the validity of the main results.
\end{abstract}

MSC: Primary 34L30; 47H11; secondary 54H25

Keywords: Discrete Nicholson's system; Variable delays; Continuation theorem; Periodic solution

\section{Introduction}

Global stability means that the attracting basin of trajectories of a dynamical system is either the state space or a certain region in the state space, which is the defining region of the state variables of the system. In other words, global stability means that any trajectories finally tend to the attractor of the system, regardless of initial conditions. For most of biological systems, population dynamics, e.g., gene regulatory systems, are needed to be globally stable [1-4]. In addition, the global asymptotical stability of the positive equilibrium of a dynamical system is one of the research foci in theoretical studies of both continuous and discrete bio-mathematical models [5-11].

Long investigated a patch structure Nicholson's blowflies model involving multiple pairs of different time-varying delays

$$
k_{i}^{\prime}(t)=-\alpha_{i i} k_{i}(t)+\sum_{j=1, j \neq i}^{n} \alpha_{i j} k_{j}(t)+\sum_{j=1}^{n} \beta_{i j}(t) k_{i}\left(t-f_{i j}(t)\right) e^{-\gamma_{i j}(t) k_{i}\left(t-g_{i j}(t)\right)}
$$

for $t \geq t_{0}, i=1,2, \ldots, n$ [11]. The author established three novel criteria to check the global convergence, generalized exponential convergence, and asymptotical stability on the zero

(c) The Author(s) 2021. This article is licensed under a Creative Commons Attribution 4.0 International License, which permits use, sharing, adaptation, distribution and reproduction in any medium or format, as long as you give appropriate credit to the original author(s) and the source, provide a link to the Creative Commons licence, and indicate if changes were made. The images or other third party material in this article are included in the article's Creative Commons licence, unless indicated otherwise in a credit line to the material. If material is not included in the article's Creative Commons licence and your intended use is not permitted by statutory regulation or exceeds the permitted use, you will need to obtain permission directly from the copyright holder. To view a copy of this licence, visit http://creativecommons.org/licenses/by/4.0/. 
equilibrium point of the addressed model, without assuming the uniform positiveness of the death rate and the boundedness of coefficients, respectively (see [11]). Zhang et al. established the existence and global exponential stability of positive almost periodic solutions for Nicholson's blowflies systems involving patch structure and nonlinear densitydependent mortality terms by applying differential inequality techniques and the fluctuation lemma which guarantees the existence of positive asymptotically almost periodic solutions for the addressed system [12]. Qian et al., under some assumptions, developed a novel approach to demonstrate the global stability of positive asymptotically almost periodic solutions for the nonlinear density-dependent mortality Nicholson's blowflies system

$$
\begin{aligned}
k_{i}^{\prime}(t)= & -a_{i i}(t)+b_{i i}(t) e^{-k_{i}(t)}+\sum_{j=1, j \neq i}^{n}\left(a_{i j}(t)-b_{i j}(t) e^{-k_{j}(t)}\right) \\
& +\sum_{j=1}^{n} \beta_{i j}(t) k_{i}\left(t-f_{i j}(t)\right) e^{-\gamma_{i j}(t) k_{i}\left(t-f_{i j}(t)\right)}
\end{aligned}
$$

for $i=1,2, \ldots, n$ [13]. Sweilam et al. considered variable-order fractional coupled nonlinear Burger's equations under proportional delay $a, b$, and $c$ in two dimensions (2-D) with the Atangana-Baleanu-Caputo (ABC) derivatives as follows:

$$
\begin{aligned}
{ }^{A B C} & \mathcal{D}_{t}^{\alpha(x, y, t)} u_{t}(x, y, t)+\lambda_{1} u(a x, b y, c t) u_{x}(x, y, c t)+\beta_{1} v(x, y, t) u_{y}(x, y, t) \\
\quad & \rho\left(u_{x x}(x, y, t)+u_{y y}(x, y, t)\right), \\
{ }^{A B C} & \mathcal{D}_{t}^{\alpha(x, y, t)} v_{t}(x, y, t)+\lambda_{2} u(a x, b y, c t) v_{x}(x, y, c t)+\beta_{2} v(x, y, t) v_{y}(x, y, t) \\
\quad & \rho\left(v_{x x}(x, y, t)+v_{y y}(x, y, t)\right),
\end{aligned}
$$

with the initial conditions $u\left(x, y, t_{0}\right)=g_{1}(x, y), v\left(x, y, t_{0}\right)=g_{2}(x, y), x, y \in\left[L_{0}, L\right]$, and some boundary conditions where $0<\alpha x, y, t) \leq 1$ and $u(x, y, t)$ and $v(x, y, t)$ are velocity components, $\rho$ is a diffusion coefficient, $\lambda_{1}, \lambda_{2}, \beta_{1}$, and $\beta_{2}$ are constants, $g_{1}(x, y), g_{2}(x, y), f_{1}(x, y, t)$, and $f_{2}(x, y, t)$ are all known functions, $t_{0}$ is the initial time, $a, b, c \in(0,1)$ [3]. Also, Sweilam et al. in [7] investigated the effect of the optimal control of the variable order for HIV/AIDS and malaria mathematical models with multi-time delay and developed an efficient numerical algorithm to approximate the solutions of the proposed model with three control variables to reduce the number of the infected individuals of malaria and HIV/AIDS, and presented the numerical simulations for the obtained variable-order fractional system. Jaradat et al. considered the effect of inherited memory time and delay time in the formulation of a mathematical population growth model

$$
\mathcal{D}_{t}^{\alpha} P(x, t)-\mathcal{D}_{x x}\left(P^{2}(x, t)\right)-a P(x, \tau t)+b P^{2}(x, \tau t)=0
$$

for $t>0$, where $\alpha \in(0,1]$ is the Caputo derivative, and introduced two different numerical schemes to study analytically the propagation of population growth [6].

In 1986, Freedman et al. introduced criteria, such as population dynamics that has always been a core topic in theoretical ecology, which are established for three classes of models of single-species dynamics with a single discrete delay to have a globally asymptotically stable positive equilibrium independent of the length of delay [5]. In fact, asymptotic 
mean square stability of the linear part of the considered equation is used to verify stability in probability of nonlinear origin equation. In biological applications, a recruitmentdelayed model

$$
\frac{\mathrm{d} k}{\mathrm{~d} t}=\mathcal{B}\left(k\left(t-t_{1}\right)\right)-\mathcal{D}(k(t))
$$

is frequently used, where $k(t)$ is a population size, the birth function $\mathcal{B}$ involves maturation delay $t_{1}$, and the death rate $\mathcal{D}$ depends on the current population level only. In order to characterize the population of the Australian sheep blowflies and to coincide well with the experimental result, Nicholson [14] and Gurney [15] introduced Nicholson's blowflies model

$$
k^{\prime}(t)=-a k(t)+b k\left(t-t_{1}\right) \exp \left(-c k\left(t-t_{1}\right)\right)
$$

where $k(t)$ denotes the population size at time $t, a$ is the per capita daily adult death rate, $b$ denotes the maximum per capita daily egg production, $\frac{1}{c}$ is the size at which the blowfly population reproduces at its maximum rate, and $t_{1}$ is delay of the generation time. In 2015, Deng et al. solved an open problem on the global attractivity of the following diffusive Nicholson's blowflies equation with distributed delay:

$$
\begin{aligned}
y_{t}-d \Delta y= & -\lambda y(x, t)+\alpha \tau\left(\int_{-\infty}^{t} w(t-\xi) y(x, \xi) \mathrm{d} \xi\right) \\
& \times \exp \left(-\int_{-\infty}^{t} w(t-\xi) y(x, \xi) \mathrm{d} \xi\right)
\end{aligned}
$$

for $(x, t) \in \Upsilon \times(0, \infty)$, where $\Upsilon$ is a bounded domain in $\mathbb{R}^{N}$ with smooth boundary $\partial \Upsilon$, the parameters $\alpha, \lambda$ are positive constants, and nonnegative kernel function $w$ satisfies $\int_{0}^{\infty} w(\xi) \mathrm{d} \xi=1$ [16]. In 2019, Gao et al. studied the conformable $(2+1)$-dimensional Ablowitz-KaupNewell-Segur equation in order to show the existence of complex combined dark-bright soliton solutions [12]. To this purpose, an effective method, i.e., the sineGordon expansion method, was used [12]. The 2D and 3D surfaces under some suitable values of parameters were also plotted [12].

Recently, many researchers have worked on a numerical technique to solve some types of equations such as Burgers' equations with proportional delay, mathematical models with multi-time delay, an SEIR epidemic model for COVID-19 transmission, and biomathematics model inherited with memory time and delay time [3, 6, 7, 17]. In 2020, Yel al. employed the sine-Gordon expansion method to shallow water wave models which are KadomtsevPetviashvili-Benjamin-Bona-Mahony and the Benney-Luke equations [18]. They constructed many new complex combined dark-bright soliton, anti-kink soliton solutions for the governing models, and the 2D, 3D, and contour plots were given under the suitable coefficients [18]. Also, García Guirao et al. applied the sine-Gordon expansion method to the extended nonlinear $(2+1)$-dimensional Boussinesq equation [10]. Many new dark, complex, and mixed dark-bright soliton solutions of the governing model have been derived [10]. Moreover, for better understanding of the results, 2D, 3D, and contour graphs under the strain conditions and the suitable values of parameters were also plotted [10]. 
In 2018, the authors considered a discrete Nicholson's blowflies model

$$
\Delta k(n)=-\alpha(n) k(n)+b(n)+\beta(n) \ln k(n-\tau(n)) \cdot \frac{k(n)}{k^{\gamma(n)}(n-\tau(n))},
$$

where $n \in \mathbb{Z}$ and $\alpha, b, \beta, \gamma, \tau: \mathbb{Z} \rightarrow[0, \infty)$ are almost periodic sequences, which involve a nonlinear density-dependent mortality term, and by using a fixed point theorem and Lyapunov functional method, obtained the existence and locally exponential stability of pseudo almost periodic solutions for the addressed Nicholson's blowflies model [19].

In [20], Berezansky et al. focused on a linear model of density-dependent mortality terms

$$
P^{\prime}(t)=a P\left(t-t_{1}\right) \exp \left(-c P\left(t-t_{1}\right)\right)-\mathcal{D}[P](t)
$$

where $\alpha>0$ and the form of function $\mathcal{D}$ may be $\mathcal{D}[P]=\frac{m P}{P+n}$ or $\mathcal{D}[P]=m-n \exp (-P)$, with positive constants $m>0$ and $n>0$. In 2017, the authors considered the non-autonomous almost periodic Nicholson's blowflies model with density-dependent mortality term of the form

$$
k^{\prime}(t)=-\frac{a(t) k(t)}{b(t)+k(t)}+p(t) k(t-\tau(t)) e^{-\beta(t) k(t-\tau(t))}
$$

where $a(t), b(t), \beta(t), p(t)$, and $\tau(t) \in C\left(\mathbb{R}, \mathbb{R}^{+}\right)$and $a(t), b(t), \beta(t), p(t), \tau(t)$ are bounded almost periodic functions [21]. Also many authors extensively studied Nicholson's blowflies model with density-dependent mortality term (see for example [22-24]). Incorporating the phenomena gives us impulsive differential systems. A lot of work has been done in this direction to research impulsive differential equations, see a few viewpoints and the references therein [25-37]. They explored a generalized form of delayed Nicholson's blowflies model with impulse

$$
\begin{cases}k^{\prime}(t)=-a k(t)+\sum_{i=1}^{n} b_{i} k\left(t-t_{1}\right) e^{-c_{i} k\left(t-t_{1}\right)}, & t \neq \eta_{m}, \\ \Delta k\left(\eta_{m}\right)=d_{m} k\left(\eta_{m}\right), & t=\eta_{m},\end{cases}
$$

to establish the existence of positive periodic solutions. In 2012, the authors considered a discrete Nicholson's blowflies model involving a linear harvesting term, and with appropriate assumptions, sufficient conditions were established for the existence and exponential convergence of positive almost periodic solutions of the model [38]. Many authors have explored the discrete Nicholson's blowflies model (for instance consider [39-42]). They derived the exponential extinction, exponential stability, exponential convergence of almost periodic and multiple periodic solutions. Furthermore, more discussions about periodic solution, global stability, and exponential stability of delayed Nicholson's blowflies model could be found in references [43-56] by using of Schauder's fixed point theorem, Krasnoselskii's fixed point theorem, and Leggett-Williams fixed point theorem. 
Here, we propose in this paper the following dual system of Nicholson's blowflies model:

$$
\left\{\begin{aligned}
k_{1}(v+1)= & k_{1}(v) \exp \left(-\frac{a_{11}(v)}{b_{11}(v)+k_{1}(v)}+\frac{a_{12}(v)}{b_{12}(v)+k_{2}(v)} \cdot \frac{k_{2}(v)}{k_{1}(v)}\right. \\
& +\frac{c_{1}(v) k_{1}\left(v-\tau_{1}(v)\right)}{k_{1}(v)} \exp \left(-\delta_{1}(v) k_{1}\left(v-\tau_{1}(v)\right)\right) \\
& \left.-\mathcal{H}_{1}(v) \frac{k_{1}\left(v-\tau_{1}(v)\right)}{k_{1}(v)}\right), \\
k_{2}(v+1)= & k_{2}(v) \exp \left(-\frac{a_{22}(v)}{b_{22}(v)+k_{2}(v)}+\frac{a_{21}(v)}{b_{21}(v)+k_{1}(v)} \cdot \frac{k_{1}(v)}{k_{2}(v)}\right. \\
& +\frac{c_{2}(v) k_{2}\left(v-\tau_{2}(v)\right)}{k_{2}(v)} \exp \left(-\delta_{2}(v) k_{2}\left(v-\tau_{2}(v)\right)\right) \\
& \left.-\mathcal{H}_{2}(v) \frac{k_{2}\left(v-\tau_{2}(v)\right)}{k_{2}(v)}\right),
\end{aligned}\right.
$$

where $a_{i j}, b_{i j}, c_{i}, \delta_{i}, \mathcal{H}_{i}: \mathbb{R} \rightarrow(0, \infty)$ for $i, j=1,2, k_{i}(t)(i=1,2)$ denotes the population's size at time $t$ and periodic functions $\tau_{1}, \tau_{2}: \mathbb{R} \rightarrow[0, \infty)$ are continuous with period $p$. By using the technical idea of Gaines and Mawhin continuation theorem of coincidence degree theory in [57], we derive the sufficient conditions for the new result of existence of positive periodic solution to system (1). Finally, one numerical simulation example is provided to verify the main results.

We arrange the rest of the paper as follows: In Sect. 2, we recall some preliminaries of the basic tool. Section 3 is devoted to showing the main results, while an example illustrating the obtained results and an algorithm for the system are presented in Sect. 4.

\section{Preliminaries}

Before exploring the existence of periodic solutions of the system, we give some denotations, which will be useful to prove the main result. Let $\mathscr{L}: \operatorname{Dom} \mathscr{L} \subset \mathcal{Y} \rightarrow \mathcal{Z}$ and $\mathscr{N}$ : $\mathcal{Y} \rightarrow \mathcal{Z}$ be a linear mapping and a continuous mapping, respectively, where $\mathcal{Y}$ and $\mathcal{Z}$ are real Banach spaces. Let $\mathcal{Y}$ and $\mathcal{Z}$ be Banach spaces and $L B(\mathcal{Y}, \mathcal{Z})$ denote the set of bounded linear operators $\mathscr{T}$ from $\mathcal{Y}$ to $\mathcal{Z}$ with $\operatorname{Dom}(\mathscr{T})=\mathcal{Y}$. An operator $\mathscr{L} \in L B(\mathcal{Y}, \mathcal{Z})$ is called a Fredholm mapping of index zero if $\operatorname{dim} \operatorname{ker} \mathscr{L}, \operatorname{codim} \operatorname{Im} \mathscr{L}$ are finite and $\operatorname{Im} \mathscr{L} \subset \mathcal{Z}$ is closed. If $\mathscr{L}$ is a Fredholm mapping of index zero, then there exist continuous projectors $\mathscr{P}: \mathcal{Y} \rightarrow \mathcal{Y}$ and $\mathscr{Q}: \mathcal{Z} \rightarrow \mathcal{Z}$ such that

$$
\operatorname{Im} \mathscr{P}=\operatorname{ker} \mathscr{L}, \quad \operatorname{ker} \mathscr{Q}=\operatorname{Im} \mathscr{L}=\operatorname{Im}(I-\mathscr{Q})
$$

It follows that the restriction $\left.\mathscr{L}\right|_{\mathscr{P}}$ of $\mathscr{L}$ to

$\operatorname{Dom} \mathscr{L} \cap \operatorname{ker} \mathscr{P}:(\mathscr{I}-\mathscr{P}) \mathcal{Y} \rightarrow \operatorname{Im} \mathscr{L}$

is invertible. Denote the inverse of $\left.\mathscr{L}\right|_{\mathscr{P}}$ by $\mathcal{K}$.

Lemma 1 ([57]) Let $\mathcal{O} \subset \mathcal{Y}$ be an open bounded set, $\mathscr{L}$ be a Fredholm mapping of index zero, and $\mathscr{N}$ be $\mathscr{L}$-compact on $\overline{\mathcal{O}}$. Assume

(I) $\mathscr{L}(z) \neq \eta \mathscr{N}(z)$ for all $\eta \in(0,1), z \in \partial \mathcal{O} \cap \operatorname{Dom} \mathscr{L}$;

(II) $\mathscr{Q}(\mathscr{N}(z)) \neq 0$ for each $z \in \partial \mathcal{O} \cap \operatorname{ker} \mathscr{L}$;

(III) $\operatorname{deg}(U \mathscr{Q} \mathscr{N}, \mathcal{O} \cap \operatorname{ker} \mathscr{L}, 0) \neq 0$.

Then $\mathscr{L}(z)=\mathscr{N}(z)$ has at least one solution in $\overline{\mathcal{O}} \cap \operatorname{Dom} \mathscr{L}$. 
Lemma 2 ([58]) Suppose that $k: \mathbb{Z} \rightarrow \mathbb{R}$ is a p-periodic function such that $k(v+p)=k(v)$.

Then, for any fixed $v_{1}, v_{2}$ belonging to

$$
I_{p}=\{0,1, \ldots, p-1\}
$$

and any $v \in \mathbb{Z}$, one has

$$
k\left(v_{2}\right)-\sum_{v=0}^{p-1}|k(v+1)-k(v)| \leq k(v) \leq k\left(v_{1}\right)+\sum_{v=0}^{p-1}|k(v+1)-k(v)| .
$$

For convenience, we shall introduce the following notations:

$$
\bar{g}=\frac{1}{p} \sum_{v=0}^{p-1} g(v), \quad g^{*}=\max _{v \in I_{p}} g(v), \quad g_{*}=\min _{v \in I_{p}} g(v) .
$$

\section{Main results}

Theorem 3 For system (1), we assume that:

(1) $a_{i j}(v), b_{i j}(v), c_{i}(v), \delta_{1}(v)$, and $\mathcal{H}_{i}(v)$ are continuous positive periodic functions with period $p>0$ and $\tau_{i}(v)$ is a nonnegative continuous function with

$$
\tau_{i}(v)=\tau_{i}(v+p),
$$

here $i, j=1,2$.

(2) $\frac{a_{11}^{*}}{b_{11} *}+\mathcal{H}_{1}^{*}>0$ and $\frac{a_{22}^{*}}{b_{22} *}+\mathcal{H}_{2}^{*}>0$.

(3) $\mathcal{H}_{1 *}>\frac{a_{12}^{*}}{b_{12 *}}$ and $\mathcal{H}_{2 *}>\frac{a_{21}^{*}}{b_{21 *}}$,

here

$$
a_{i j}^{*}=\max _{\nu \in I_{p}} a_{i j}(v), \quad b_{i j_{*}}=\min _{v \in I_{p}} b_{i j}(v), \quad(i, j=1,2)
$$

and

$$
\mathcal{H}_{i}^{*}=\max _{v \in I_{p}} \mathcal{H}_{i}(v), \quad \mathcal{H}_{i *}=\min _{v \in I_{p}} \mathcal{H}_{i}(v) .
$$

Then there exists at least one positive periodic solution of system (1).

Proof By the biological meaning, we only focus on the positive periodic solutions to system (1). Let the transformation be $k_{1}(v)=\exp \left(\varrho_{1}(v)\right)$ and $k_{2}(v)=\exp \left(\varrho_{2}(v)\right)$. Then system (1) becomes

$$
\left\{\begin{array}{l}
\varrho_{1}(v+1)-\varrho_{1}(v)=\theta_{1}(v) \\
\varrho_{2}(v+1)-\varrho_{2}(v)=\theta_{2}(v)
\end{array}\right.
$$

where

$$
\theta_{1}(v)=-\frac{a_{11}(v)}{b_{11}(v)+\exp \left(\varrho_{1}(v)\right)}
$$




$$
\begin{aligned}
& +\frac{a_{12}(v)}{b_{12}(v)+\exp \left(\varrho_{2}(v)\right)} \exp \left(\varrho_{2}(v)-\varrho_{1}(v)\right) \\
& -\mathcal{H}_{1}(v) \exp \left(\varrho_{1}\left(v-\tau_{1}(v)\right)-\varrho_{1}(v)\right) \\
& +c_{1}(v) \exp \left(\varrho_{1}\left(v-\tau_{1}(v)\right)-\varrho_{1}(v)-\delta_{1}(v) \exp \left(\varrho_{1}\left(v-\tau_{1}(v)\right)\right)\right), \\
\theta_{2}(v)= & -\frac{a_{22}(v)}{b_{22}(v)+\exp \left(\varrho_{2}(v)\right)} \\
& +\frac{a_{21}(v)}{b_{21}(v)+\exp \left(\varrho_{1}(v)\right)} \exp \left(\varrho_{1}(v)-\varrho_{2}(v)\right) \\
& -\mathcal{H}_{2}(v) \exp \left(\varrho_{2}\left(v-\tau_{2}(v)\right)-\varrho_{2}(v)\right) \\
& +c_{2}(v) \exp \left(\varrho_{2}\left(v-\tau_{2}(v)\right)-\varrho_{2}(v)-\delta_{2}(v) \exp \left(\varrho_{2}\left(v-\tau_{2}(v)\right)\right)\right) .
\end{aligned}
$$

Since (4) has a $p$-periodic solution $\left(\varrho_{1}(v), \varrho_{2}(v)\right)^{T}$, it is easy to see that

$$
\left(k_{1}(v), k_{2}(v)\right)^{T}=\left(\exp \left(\varrho_{1}(v)\right), \exp \left(\varrho_{2}(l)\right)\right)^{T}
$$

is a positive $p$-periodic solution of (1). Next, it needs to show that (4) has a $p$-periodic solution. Define

$$
j_{2}=\left\{z=(z(v)): z(v) \in \mathbb{R}^{2}, v \in \mathbb{Z}\right\} .
$$

For $r=\left(r_{1}, r_{2}\right)^{T} \in \mathbb{R}^{2}$, define $|r|=\max \left\{\left|r_{1}\right|,\left|r_{2}\right|\right\}$. Let $j^{p} \subset j_{2}$ denote the subspace of all $p$ periodic sequences equipped with the usual supremum norm $\|\cdot\|$, i.e.,

$$
\|z\|=\max _{\nu \in I_{p}}|z(v)|
$$

for any

$$
z=\{z(v): v \in \mathbb{Z}\} \in j^{p} .
$$

It is obvious that $j^{p}$ is a finite dimensional Banach space. Let

$$
j_{0}^{p}=\left\{z=(z(v)) \in j^{p}: \sum_{l=0}^{p-1} z(v)=0\right\}
$$

and

$$
j_{c}^{p}=\left\{z=(z(v)) \in j^{p}: z(v)=h \in \mathbb{R}^{2}, v \in \mathbb{Z}\right\} .
$$

Then it follows that $j_{0}^{p}$ and $j_{c}^{p}$ are both closed linear subspaces of $j^{p}$ and $j^{p}=j_{c}^{p} \oplus j_{0}^{p}$, $\operatorname{dim} j_{c}^{p}=2$. We take

$$
j^{p}=\mathcal{Z}=\left\{z(v)=\left(\varrho_{1}(v), \varrho_{2}(v)\right)^{T} \in \mathbb{R}^{2}: \varrho_{1}(v+p)=\varrho_{1}(v), \varrho_{2}(v+p)=\varrho_{2}(v)\right\}
$$

and

$$
\|z\|=\left\|\left(\varrho_{1}(v), \varrho_{2}(v)\right)^{T}\right\|=\max _{v \in I_{p}}\left|\varrho_{1}(v)\right|+\max _{v \in I_{p}}\left|\varrho_{2}(v)\right| .
$$


Then $\mathcal{Z}$ is a Banach space with norm $\|\cdot\|$. Now, we define $\mathscr{L}: \operatorname{Dom}(\mathscr{L}) \subset \mathcal{Z} \rightarrow \mathcal{Z}$ and $\mathscr{N}: \mathcal{Z} \rightarrow \mathcal{Z}$ by

$$
\mathscr{L}\left(\varrho_{1}, \varrho_{2}\right)=\left(\begin{array}{l}
\varrho_{1}(v+1)-\varrho_{1}(v) \\
\varrho_{2}(v+1)-\varrho_{2}(v)
\end{array}\right)
$$

and

$$
\mathscr{N}\left(\varrho_{1}, \varrho_{2}\right)=\left(\begin{array}{l}
\theta_{1}(v) \\
\theta_{2}(v)
\end{array}\right)
$$

for any $\left(\varrho_{1}, \varrho_{2}\right)^{T} \in \mathcal{Z}$ and $v \in \mathbb{Z}$, here $\theta_{1}(v)$ and $\theta_{2}(v)$ are defined by Eq. (5). It is trivial to get the argument $\mathscr{L}$ is a bounded linear operator, ker $\mathscr{L}=j_{c}^{p}, \operatorname{Im} \mathscr{L}=j_{0}^{p}$, and $\operatorname{Im} \mathscr{L} \subset \mathcal{Z}$ is closed. Therefore, $\operatorname{dim} \operatorname{ker} \mathscr{L}=\operatorname{codim} \operatorname{Im} \mathscr{L}=2$. Indeed, $\mathscr{L}$ is a Fredholm mapping of index zero. At present, we set the continuous projectors $\mathscr{P}: \mathcal{Z} \rightarrow \mathcal{Z}$ and $\mathscr{Q}: \mathcal{Z} \rightarrow \mathcal{Z}$ defined by

$$
\mathscr{P}\left(\varrho_{1}, \varrho_{2}\right)=\mathscr{Q}\left(\varrho_{1}, \varrho_{2}\right)=\left(\begin{array}{l}
\frac{1}{p}\left[\sum_{v=0}^{p-1} \varrho_{1}(v)\right] \\
\frac{1}{p}\left[\sum_{v=0}^{p-1} \varrho_{2}(v)\right]
\end{array}\right),
$$

such that Eq. (2) holds. Furthermore, $\mathcal{K}$ denotes the inverse of $\left.\mathscr{L}\right|_{\text {Dom } \mathscr{K} \cap \text { ker } \mathscr{P}}$,

$$
\mathcal{K}\left(\varrho_{1}, \varrho_{2}\right)=\left(\begin{array}{l}
\sum_{v=0}^{p-1} \varrho_{1}(v)-\frac{1}{p}\left[\sum_{v=0}^{p-1}(p-v) \varrho_{1}(v)\right] \\
\sum_{v=0}^{p-1} \varrho_{2}(v)-\frac{1}{p}\left[\sum_{v=0}^{p-1}(p-v) \varrho_{2}(v)\right]
\end{array}\right),
$$

$\mathscr{Q} \mathscr{N}: \mathcal{Z} \rightarrow \mathcal{Z}$ and $\mathcal{K}(I-\mathscr{Q}) \mathscr{N}: \mathcal{Z} \rightarrow \mathcal{Z}$ are defined by

$$
\begin{aligned}
& \mathscr{Q} \mathscr{N}\left(\varrho_{1}, \varrho_{2}\right)=\left(\begin{array}{c}
\frac{1}{p} \sum_{v=0}^{p-1} \theta_{1}(v) \\
\frac{1}{p} \sum_{v=0}^{p-1} \theta_{2}(v)
\end{array}\right), \\
& \mathcal{K}(I-\mathscr{Q}) \mathscr{N}\left(\varrho_{1}, \varrho_{2}\right)=\left(\begin{array}{l}
\sum_{v=0}^{p-1} \theta_{1}(v) \\
\sum_{v=0}^{p-1} \theta_{2}(v)
\end{array}\right)-\left(\begin{array}{c}
\frac{1}{p} \sum_{v=0}^{p-1}(p-v) \theta_{1}(v) \\
\frac{1}{p} \sum_{v=0}^{p-1}(p-v) \theta_{2}(v)
\end{array}\right) \\
&-\left(\begin{array}{c}
\left(\frac{v}{p}-\frac{p+1}{2 p}\right) \sum_{v=0}^{p-1} \theta_{1}(v) \\
\left(\frac{v}{p}-\frac{p+1}{2 p}\right) \sum_{v=0}^{p-1} \theta_{2}(v)
\end{array}\right) .
\end{aligned}
$$

This implies that functions $\mathscr{Q} \mathscr{N}$ and $\mathcal{K}(I-\mathscr{Q}) \mathscr{N}$ are all continuous. On the other hand, $\mathcal{Z}$ is a finite dimensional Banach space, and so the Arzelà-Ascoli theorem implies that

$$
\overline{\mathcal{K}(I-\mathscr{Q}) \mathscr{N}(\overline{\mathcal{O}})}
$$

is compact for any open bounded set $\mathcal{O} \subset \mathcal{Z}$. Moreover, $\mathscr{Q} \mathscr{N}(\overline{\mathcal{O}})$ is bounded. Therefore, $\mathscr{N}$ is $\mathscr{L}$-compact on $\overline{\mathcal{O}}$ for any open bounded set $\mathcal{O} \subset \mathcal{Z}$. The isomorphism $J: \operatorname{Im} \mathscr{Q} \rightarrow$ $\operatorname{ker} \mathscr{L}$ is an identity mapping such that $\operatorname{ker} \mathscr{L}=\operatorname{Im} \mathscr{Q}$. In the following, we consider the operator equation $\mathscr{L} \varrho=\eta \mathscr{N} \varrho$ for $\eta \in(0,1)$, that is,

$$
\left\{\begin{array}{l}
\varrho_{1}(v+1)-\varrho_{1}(v)=\eta \theta_{1}(v), \\
\varrho_{2}(v+1)-\varrho_{2}(v)=\eta \theta_{2}(v) .
\end{array}\right.
$$


Suppose that $\left(\varrho_{1}(v), \varrho_{2}(v)\right)^{T} \in \mathcal{Z}$ is a solution of (6) for certain $\eta \in(0,1)$, and summing from 0 to $p-1$ on both sides, we get

$$
\left\{\begin{array}{l}
\sum_{v=0}^{p-1} \theta_{1}(v)=0, \\
\sum_{v=0}^{p-1} \theta_{2}(v)=0 .
\end{array}\right.
$$

Combining the first equation of system (6) and the first equation of system (7), we have

$$
\sum_{\nu=0}^{p-1}\left|\varrho_{1}(v+1)-\varrho_{1}(v)\right|<M_{1},
$$

here

$$
M_{1}=2 \sum_{\nu=0}^{p-1} \frac{a_{11}(v)}{b_{11}(v)} .
$$

From the second equation of system (6) and the second equation of system (7), we have

$$
\sum_{v=0}^{p-1}\left|\varrho_{2}(v+1)-\varrho_{2}(v)\right|<M_{2},
$$

here

$$
M_{2}=2 \sum_{v=0}^{p-1} \frac{a_{22}(v)}{b_{22}(v)} .
$$

Multiplying the first equation of system (7) by $\exp \left(\varrho_{1}(l)\right)$, we obtain

$$
\begin{aligned}
\sum_{v=0}^{p-1} & \frac{a_{11}(v)}{b_{11}(v)+\exp \left(\varrho_{1}(v)\right)} \exp \left(\varrho_{1}(v)\right)+\sum_{v=0}^{p-1} \mathcal{H}_{1}(v) \exp \left(\varrho_{1}\left(v-\tau_{1}(v)\right)\right) \\
= & \sum_{v=0}^{p-1} \frac{a_{12}(v)}{b_{12}(v)+\exp \left(\varrho_{2}(v)\right)} \exp \left(\varrho_{2}(v)\right) \\
& +\sum_{v=0}^{p-1} c_{1}(v) \exp \left(\varrho_{1}\left(v-\tau_{1}(v)\right)-\delta_{1}(v) e^{\varrho_{1}\left(v-\tau_{1}(v)\right)}\right) .
\end{aligned}
$$

Notice that

$$
\begin{aligned}
& \sum_{v=0}^{p-1} \exp \left(\varrho_{1}\left(v-\tau_{1}(v)\right)\right)=\sum_{\nu=0}^{p-1} \exp \left(\varrho_{1}(v)\right), \\
& \sum_{v=0}^{p-1} \exp \left(\varrho_{2}\left(v-\tau_{2}(v)\right)\right)=\sum_{\nu=0}^{p-1} \exp \left(\varrho_{2}(v)\right)
\end{aligned}
$$

and

$$
\sup _{\varrho \geq 0} \varrho \exp (-\varrho)=\frac{1}{\exp (1)} .
$$


Since $\left(\varrho_{1}(v), \varrho_{2}(v)\right)^{T} \in \mathcal{Z}$, there exist $\alpha_{i}, \beta_{i} \in I_{p}$ such that

$$
\varrho_{i}\left(\alpha_{i}\right)=\max _{\nu \in I_{p}} \varrho_{i}(\nu), \quad \varrho_{i}\left(\beta_{i}\right)=\min _{\nu \in I_{p}} \varrho_{i}(\nu)
$$

with $i=1,2$. Substituting (11), (12), and (13) in (10), we get

$$
\begin{aligned}
& \sum_{\nu=0}^{p-1} \frac{a_{11}(\nu)}{b_{11}(\nu)+\exp \left(\varrho_{1}(\nu)\right)} \exp \left(\varrho_{1}(\nu)\right)+\sum_{\nu=0}^{p-1} \mathcal{H}_{1}(\nu) \exp \left(\varrho_{1}(\nu)\right) \\
& =\sum_{\nu=0}^{p-1} \frac{a_{12}(v)}{b_{12}(v)+\exp \left(\varrho_{2}(v)\right)} \exp \left(\varrho_{2}(v)\right) \\
& +\sum_{\nu=0}^{p-1} \frac{c_{1}(v)}{\delta_{1}(v)} \delta_{1}(v) \exp \left(\varrho_{1}\left(v-\tau_{1}(v)\right)-\delta_{1}(v) \exp \left(\varrho_{1}\left(v-\tau_{1}(v)\right)\right)\right), \\
& {\left[\frac{a_{11}^{*}}{b_{11 *}}+\mathcal{H}_{1}^{*}\right] \sum_{\nu=0}^{p-1} \exp \left(\varrho_{1}(\nu)\right)>\sum_{\nu=0}^{p-1} \frac{c_{1}(v)}{\delta_{1}(\nu) \exp (1)},} \\
& {\left[\frac{a_{11} *}{b_{11 *}}+\mathcal{H}_{1}^{*}\right] \exp \left(\varrho_{1}\left(\alpha_{1}\right)\right)>\frac{c_{1 *}}{\delta_{1}^{*} \exp (1)}}
\end{aligned}
$$

and

$$
\varrho_{1}\left(\alpha_{1}\right)>\log \left[\frac{c_{1 *} b_{11 *}}{\delta_{1}^{*} \exp (1)\left(a_{11}{ }^{*}+b_{11 *} \mathcal{H}_{1}{ }^{*}\right)}\right] .
$$

Multiplying the second equation of system (7) by $\exp \left(\varrho_{2}(v)\right)$, we obtain

$$
\begin{aligned}
& \sum_{\nu=0}^{p-1} \frac{a_{22}(v)}{b_{22}(v)+\exp \left(\varrho_{2}(v)\right)} \exp \left(\varrho_{2}(v)\right)+\sum_{\nu=0}^{p-1} \mathcal{H}_{2}(v) \exp \left(\varrho_{2}\left(v-\tau_{2}(v)\right)\right) \\
& =\sum_{\nu=0}^{p-1} \frac{a_{21}(v)}{b_{21}(v)+\exp \left(\varrho_{1}(v)\right)} \exp \left(\varrho_{1}(v)\right) \\
& \quad+\sum_{\nu=0}^{p-1} c_{2}(v) \exp \left(\varrho_{2}\left(v-\tau_{2}(v)\right)-\delta_{2}(v) \exp \left(\varrho_{2}\left(v-\tau_{2}(v)\right)\right)\right) .
\end{aligned}
$$

Substituting Eqs. (11), (12), and (13) in (15), we get

$$
\begin{aligned}
& \sum_{\nu=0}^{p-1} \frac{a_{22}(v)}{b_{22}(v)+\exp \left(\varrho_{2}(v)\right)} \exp \left(\varrho_{2}(v)\right)+\sum_{\nu=0}^{p-1} \mathcal{H}_{2}(v) \exp \left(\varrho_{2}(v)\right) \\
& =\sum_{\nu=0}^{p-1} \frac{a_{21}(v)}{b_{21}(v)+\exp \left(\varrho_{1}(v)\right)} \exp \left(\varrho_{1}(\nu)\right) \\
& +\sum_{v=0}^{p-1} \frac{c_{2}(v)}{\delta_{2}(v)} \delta_{2}(v) \exp \left(\varrho_{2}\left(v-\tau_{2}(v)\right)-\delta_{2}(v) \exp \left(\varrho_{2}\left(v-\tau_{2}(v)\right)\right)\right), \\
& {\left[\frac{a_{22}{ }^{*}}{b_{22}}+\mathcal{H}_{2} *\right] \sum_{\nu=0}^{p-1} \exp \left(\varrho_{2}(\nu)\right)>\sum_{\nu=0}^{p-1} \frac{c_{2}(\nu)}{\delta_{2}(\nu) \exp (1)},}
\end{aligned}
$$




$$
\left[\frac{a_{22}{ }^{*}}{b_{22 *}}+\mathcal{H}_{2}{ }^{*}\right] \exp \left(\varrho_{2}\left(\alpha_{2}\right)\right)>\frac{c_{2 *}}{\delta_{2}^{*} \exp (1)}
$$

and

$$
\varrho_{2}\left(\eta_{2}\right)>\log \left[\frac{c_{2 *} b_{22 *}}{\delta_{2}^{*} \exp (1)\left(a_{22}{ }^{*}+b_{22 *} \mathcal{H}_{2}{ }^{*}\right)}\right] .
$$

From Eqs. (10) and (11), we obtain

$$
\begin{aligned}
\sum_{v=0}^{p-1} \mathcal{H}_{1}(v) \exp \left(\varrho_{1}(v)\right)< & \sum_{v=0}^{p-1} \frac{a_{12}(v)}{b_{12}(v)+\exp \left(\varrho_{2}(v)\right)} \exp \left(\varrho_{2}(v)\right) \\
& +\sum_{v=0}^{p-1} c_{1}(v) \exp \left(\varrho_{1}\left(v-\tau_{1}(v)\right)-\delta_{1}(v)\right. \\
& \left.\times \exp \left(\varrho_{1}\left(v-\tau_{1}(v)\right)\right)\right) .
\end{aligned}
$$

If

$$
\sum_{\nu=0}^{p-1} \exp \left(\varrho_{2}(v)\right) \leq \sum_{v=0}^{p-1} \exp \left(\varrho_{1}(v)\right)
$$

and (12), then it follows from Eqs. (13) and (17) that

$$
\begin{gathered}
\sum_{v=0}^{p-1} \mathcal{H}_{1}(v) \exp \left(\varrho_{1}(v)\right)<\sum_{v=0}^{p-1} \frac{a_{12}(v)}{b_{12}(v)+\exp \left(\varrho_{2}(v)\right)} \exp \left(\varrho_{1}(v)\right) \\
+\sum_{v=0}^{p-1} c_{1}(v) \exp \left(\varrho_{1}\left(v-\tau_{1}(v)\right)-\delta_{1}(v)\right. \\
\left.\times \exp \left(\varrho_{1}\left(v-\tau_{1}(v)\right)\right)\right) \\
{\left[H_{1 *}-\frac{a_{12}}{b_{12 *}}\right] \sum_{v=0}^{p-1} \exp \left(\varrho_{2}(v)\right) \leq\left[\mathcal{H}_{1 *}-\frac{a_{12}}{b_{12 *}}\right] \sum_{v=0}^{p-1} \exp \left(\varrho_{1}(v)\right)} \\
<\sum_{v=0}^{p-1} \frac{c_{1}(v)}{\delta_{1}(v) \exp (1)}
\end{gathered}
$$

and

$$
\varrho_{2}\left(\beta_{2}\right)<\log \left[\frac{c_{1}{ }^{*} b_{12 *}}{\delta_{1 *} \exp (1)\left(b_{12 *} \mathcal{H}_{1 *}-a_{12}{ }^{*}\right)}\right] .
$$

From (11) and (15) we obtain

$$
\begin{aligned}
\sum_{v=0}^{p-1} \mathcal{H}_{2}(v) \exp \left(\varrho_{2}(v)\right)< & \sum_{v=0}^{p-1} \frac{a_{21}(v)}{b_{21}(v)+\exp \left(\varrho_{1}(v)\right)} \exp \left(\varrho_{1}(v)\right) \\
& +\sum_{v=0}^{p-1} c_{2}(v) \exp \left(\varrho_{2}\left(v-\tau_{2}(v)\right)-\delta_{2}(v) \exp \left(\varrho_{2}\left(v-\tau_{2}(v)\right)\right)\right)
\end{aligned}
$$


If

$$
\sum_{\nu=0}^{p-1} \exp \left(\varrho_{1}(v)\right) \leq \sum_{\nu=0}^{p-1} \exp \left(\varrho_{2}(v)\right)
$$

and (12), then from (13) and (19) we obtain

$$
\begin{gathered}
\sum_{v=0}^{p-1} \mathcal{H}_{2}(v) \exp \left(\varrho_{2}(v)\right)<\sum_{v=0}^{p-1} \frac{a_{21}(v)}{b_{21}(v)+\exp \left(\varrho_{1}(v)\right.} \exp \left(\varrho_{2}(v)\right) \\
+\sum_{v=0}^{p-1} c_{2}(v) \exp \left(\varrho_{2}\left(v-\tau_{2}(v)\right)-\delta_{2}(v)\right. \\
\left.\times \exp \left(\varrho_{2}\left(v-\tau_{2}(v)\right)\right)\right), \\
{\left[\mathcal{H}_{2 *}-\frac{a_{21}}{b_{21 *}}\right] \sum_{v=0}^{p-1} \exp \left(\varrho_{1}(v)\right) \leq\left[\mathcal{H}_{2 *}-\frac{a_{21} *}{b_{21}}\right] \sum_{v=0}^{p-1} \exp \left(\varrho_{2}(v)\right)} \\
<\sum_{v=0}^{p-1} \frac{c_{2}(v)}{\delta_{2}(v) \exp (1)},
\end{gathered}
$$

and

$$
\varrho_{1}\left(\beta_{1}\right)<\log \left[\frac{c_{2}{ }^{*} b_{21 *}}{\delta_{2 *} \exp (1)\left(b_{21 *} \mathcal{H}_{2 *}-a_{21}{ }^{*}\right)}\right] .
$$

From Lemma (2), (8), (9), (14), and (16), we obtain

$$
\left\{\begin{aligned}
\varrho_{1}(v) & \geq \varrho_{1}\left(\alpha_{1}\right)-\sum_{v=0}^{p-1}\left|\varrho_{1}(v+1)-\varrho_{1}(v)\right| \\
& >\log \left[\frac{c_{1 *} b_{11 *}}{\delta_{1}^{*} \exp (1)\left(a_{11}{ }^{*}+b_{11 *} \mathcal{H}_{1}{ }^{*}\right)}\right]-M_{1}, \\
\varrho_{2}(v) & \geq \varrho_{1}\left(\alpha_{2}\right)-\sum_{v=0}^{p-1}\left|\varrho_{2}(v+1)-\varrho_{2}(v)\right| \\
& >\log \left[\frac{c_{2 *} b_{22 *}}{\delta_{2}^{*} \exp (1)\left(a_{22}{ }^{*}+b_{22 *} \mathcal{H}_{2}{ }^{*}\right)}\right]-M_{2} .
\end{aligned}\right.
$$

From Lemma 2, equations (8), (9), (18), and (20), we get

$$
\left\{\begin{aligned}
\varrho_{1}(v) & \leq \varrho_{1}\left(\beta_{1}\right)+\sum_{v=0}^{p-1}\left|\varrho_{1}(v+1)-\varrho_{1}(v)\right| \\
& <\log \left[\frac{c^{*} b_{21 *}}{\delta_{2 *} \exp (1)\left(b_{21 *} \mathcal{H}_{2 *}-a_{21}{ }^{*}\right)}\right]+M_{1}, \\
\varrho_{2}(v) & \leq \varrho_{2}\left(\beta_{2}\right)+\sum_{v=0}^{p-1}\left|\varrho_{2}(v+1)-\varrho_{2}(v)\right| \\
& <\log \left[\frac{c^{*} b_{12 *}}{\delta_{1 *} \exp (1)\left(b_{12 *} \mathcal{H}_{1 *}-a_{12}{ }^{*}\right)}\right]+M_{2} .
\end{aligned}\right.
$$

From the first equations of (21) and (22), we have $\max _{v \in I_{p}}\left|\varrho_{1}(v)\right|<S_{1}$, where

$$
\begin{gathered}
S_{1}=\max \left\{\left|\log \left[\frac{c_{1 *} b_{11 *}}{\delta_{1}^{*} \exp (1)\left(a_{11}{ }^{*}+b_{11 *} \mathcal{H}_{1}{ }^{*}\right)}\right]\right|+M_{1},\right. \\
\left.\left|\log \left[\frac{c_{2}{ }^{*} b_{21 *}}{\delta_{2 *} \exp (1)\left(b_{21 *} \mathcal{H}_{2 *}-a_{21}{ }^{*}\right)}\right]\right|+M_{1}\right\} .
\end{gathered}
$$


By a similar argument, the second equations of (21) and (22) imply that $\max _{v \in I_{p}}\left|\varrho_{2}(v)\right|<$ $S_{2}$, where

$$
\begin{gathered}
S_{2}=\max \left\{\left|\log \left[\frac{c_{2 *} b_{22 *}}{\delta_{2}^{*} \exp (1)\left(a_{22}{ }^{*}+b_{22 *} \mathcal{H}_{2}^{*}\right)}\right]\right|+M_{2},\right. \\
\left.\left|\log \left[\frac{c_{1}{ }^{*} b_{12 *}}{\delta_{1 *} \exp (1)\left(b_{12 *} \mathcal{H}_{1 *}-a_{12}{ }^{*}\right)}\right]\right|+M_{2}\right\} .
\end{gathered}
$$

Clearly, $S_{1}$ and $S_{2}$ are independent of $\eta$. Denote $S=S_{1}+S_{2}+S_{0}$, where $S_{0}$ is sufficiently large such that each solution $\left(\varrho_{1}, \varrho_{2}\right)^{T}$ of the system of algebraic equations

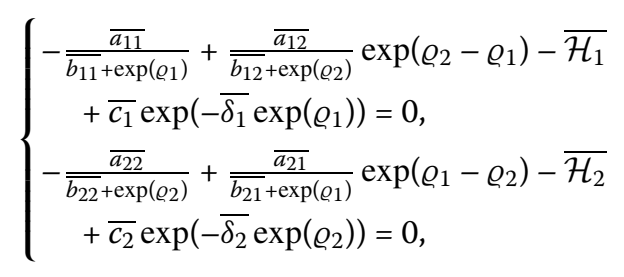

satisfies $\left\|\left(\varrho_{1}, \varrho_{2}\right)\right\|=\left|\varrho_{1}\right|+\left|\varrho_{2}\right|<S$ and $\max \left|\varrho_{1}(v)\right|+\max \left|\varrho_{2}(v)\right|<S$. Define a set as follows:

$$
\mathcal{O}=\left\{\left(\varrho_{1}(v), \varrho_{2}(v)\right)^{T} \in \mathcal{Z}:\left\|\left(\varrho_{1}, \varrho_{2}\right)^{T}\right\|<S\right\}
$$

This satisfies condition (I) in Lemma 1. If

$$
\varrho \in \partial \mathcal{O} \cap \operatorname{ker} \mathscr{L}=\partial \mathcal{O} \cap \mathbb{R}^{2},
$$

then $\varrho$ is a constant vector in $\mathbb{R}^{2}$ with $\|\varrho\|=S$ satisfying

$$
\mathscr{Q} \mathscr{N}\left(\varrho_{1}, \varrho_{2}\right)=\left(\begin{array}{c}
-\frac{\overline{a_{11}}}{\overline{b_{11}}+\exp \left(\varrho_{1}\right)}+\frac{\overline{a_{12}}}{\overline{b_{12}}+\exp \left(\varrho_{2}\right)} \exp \left(\varrho_{2}-\varrho_{1}\right) \\
-\overline{\mathcal{H}_{1}}+\overline{c_{1}} \exp \left(-\overline{\delta_{1}} \exp \left(\varrho_{1}\right)\right) \\
-\frac{\overline{a_{22}}}{\overline{b_{22}}+\exp \left(\varrho_{2}\right)}+\frac{\overline{a_{21}}}{\overline{b_{21}}+\exp \left(\varrho_{1}\right)} \exp \left(\varrho_{1}-\varrho_{2}\right) \\
-\overline{\mathcal{H}_{2}}+\overline{c_{2}} \exp \left(-\overline{\delta_{2}} e^{\varrho_{2}}\right)
\end{array}\right) \neq\left(\begin{array}{l}
0 \\
0
\end{array}\right) .
$$

Therefore condition (II) is satisfied in Lemma 1. In order to verify condition (III) in Lemma 1 , we consider a homotopy

$$
B_{\mu}\left(\left(\varrho_{1}, \varrho_{2}\right)^{T}\right)=\mu J \mathscr{Q} \mathscr{N}\left(\left(\varrho_{1}, \varrho_{2}\right)^{T}\right)+(1-\mu) \rho\left(\left(\varrho_{1}, \varrho_{2}\right)^{T}\right) .
$$

By a direct computation and the invariance property of homotopy, one has

$$
\begin{aligned}
& \operatorname{deg}\left(J \mathscr{Q} \mathscr{N}\left(\varrho_{1}, \varrho_{2}\right)^{T}, \Omega \cap \operatorname{ker} \mathscr{L},(0,0)^{T}\right) \\
& \quad=\operatorname{deg}\left(\rho\left(\varrho_{1}, \varrho_{2}\right)^{T}, \mathcal{O} \cap \operatorname{ker} \mathscr{Q},(0,0)^{T}\right) \neq 0 .
\end{aligned}
$$

Hence $\Omega$ verifies all the requirements in Lemma 1. Then we get that equation (4) has at least one periodic solution $\left(\varrho_{1}, \varrho_{2}\right)^{T}$ with period $p$ in Dom $\mathscr{Q} \cap \mathcal{O}$, which implies that system (1) has at least one positive periodic solution

$$
\left(\exp \left(\varrho_{1}\right), \exp \left(\varrho_{2}\right)\right)^{T}
$$

with period $p$. The proof is completed. 


\section{An example for the system}

Now, we illustrate the main Theorem 3 with the following model.

Example 1 Consider that a discrete Nicholson's dual system similar to system (1)

$$
\left\{\begin{aligned}
k_{1}(v+1)= & k_{1}(v) \exp \left(-\frac{0.8+0.1 \cos v}{0.7+0.3 \sin v+k_{1}(v)}\right. \\
& +\frac{0.6+0.1 \cos v}{0.9+0.2 \sin v+k_{2}(v)} \frac{k_{2}(v)}{k_{1}(v)} \\
& -(2.2+\cos v) \frac{k_{1}(v-(0.8+0.2 \cos v))}{k_{1}(v)} \\
& +\frac{(1.6+0.3 \cos v) k_{1}(v-(0.8+0.2 \cos v))}{k_{1}(v)} \\
& \left.\times \exp \left(-\left(1+\frac{\sin v}{2}\right) k_{1}(v-(0.8+0.2 \cos v))\right)\right) \\
k_{2}(v+1)= & k_{2}(v) \exp \left(-\frac{0.7+0.3 \cos v}{0.8+0.1 \sin v+k_{2}(v)}\right. \\
& +\frac{0.9+0.2 \cos v}{0.6+0.1 \sin v+k_{1}(v)} \frac{k_{1}(v)}{k_{2}(v)} \\
& -(3-0.1 \sin v) \frac{k_{2}(v-(0.6+0.1 \sin v))}{k_{2}(v)} \\
& +\frac{(1.4+0.1 \cos v) k_{2}(v-(0.6+0.1 \sin v))}{k_{2}(v)} \\
& \left.\times \exp \left(-\left(1+\frac{\cos v}{2}\right) k_{2}(v-(0.6+0.1 \sin v))\right)\right)
\end{aligned}\right.
$$

has at least one $2 \pi$-periodic solutions. Clearly, $I_{p}=\{0,1,2, \ldots, 19,20\}$ and

$$
\begin{aligned}
& a_{i j}(l)=\left[\begin{array}{ll}
0.8+0.1 \cos l & 0.6+0.1 \cos l \\
0.9+0.2 \cos l & 0.7+0.3 \cos l
\end{array}\right], \\
& b_{i j}(l)=\left[\begin{array}{ll}
0.7+0.3 \sin l & 0.9+0.2 \sin l \\
0.6+0.1 \sin l & 0.8+0.1 \sin l
\end{array}\right] \text {, } \\
& c_{i}(v)=\left[\begin{array}{l}
1.6+0.3 \cos v \\
1.4+0.1 \cos v
\end{array}\right], \quad \tau_{i}(v)=\left[\begin{array}{c}
0.8+0.2 \cos v \\
0.6+0.1 \sin v
\end{array}\right] \text {, } \\
& \delta_{i}(v)=\left[\begin{array}{c}
1+\frac{\sin v}{2} \\
1+\frac{\cos v}{2}
\end{array}\right], \quad \mathcal{H}_{i}(v)=\left[\begin{array}{c}
2.2+\cos v \\
3.0-0.1 \sin v
\end{array}\right]
\end{aligned}
$$

for $i, j=1,2$. Table 1 shows the results of $a_{i j}$ and $b_{i j}$, where $i=1,2$ and $j=1,2$. Also, one can see the graphs of $a_{i j}$ and $b_{i j}$ in Figs. 1 and 2 . In addition, for $v \in I_{p}$, we get

$$
\begin{array}{ll}
a_{11}^{*}=0.9000, & a_{12}^{*}=0.7000, \quad a_{21}^{*}=1.1000, \quad a_{22}^{*}=1.0000, \\
b_{11 *}=0.4123, & b_{12 *}=0.7082, \quad b_{21 *}=0.5041, \quad b_{22 *}=0.7041 .
\end{array}
$$

Now, by using (3), we obtain

$$
\mathcal{H}_{1}^{*}=3.5000, \quad \mathcal{H}_{2}^{*}=3.0958, \quad \mathcal{H}_{1 *}=1.2100, \quad \mathcal{H}_{2 *}=2.9090 .
$$

In this level, the numerical results in Table 1 imply that

$$
\frac{a_{11}^{*}}{b_{11} *}+\mathcal{H}_{1}^{*}=5.3827>0, \quad \frac{a_{22}^{*}}{b_{22} *}+\mathcal{H}_{2}^{*}=4.5161>0,
$$




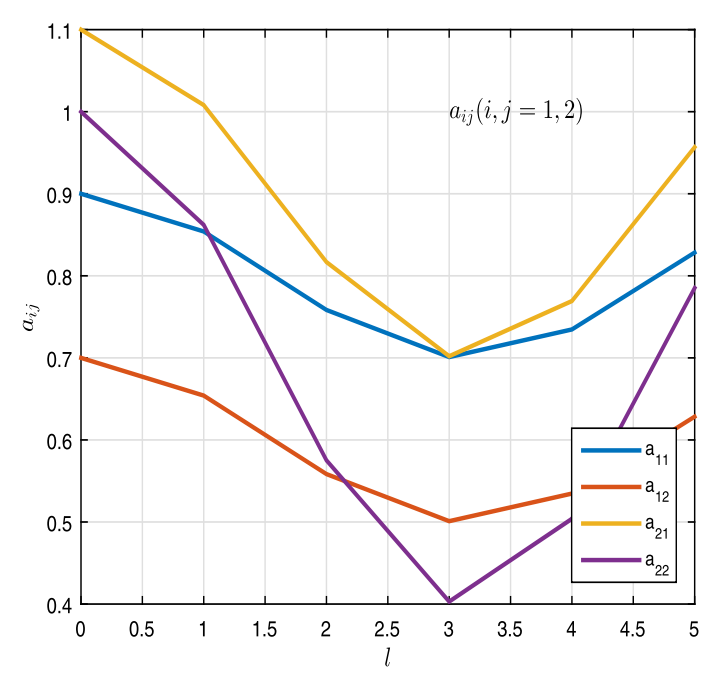

Figure 1 Graphics of $a_{i j}, i, j=1,2$, in Example 1

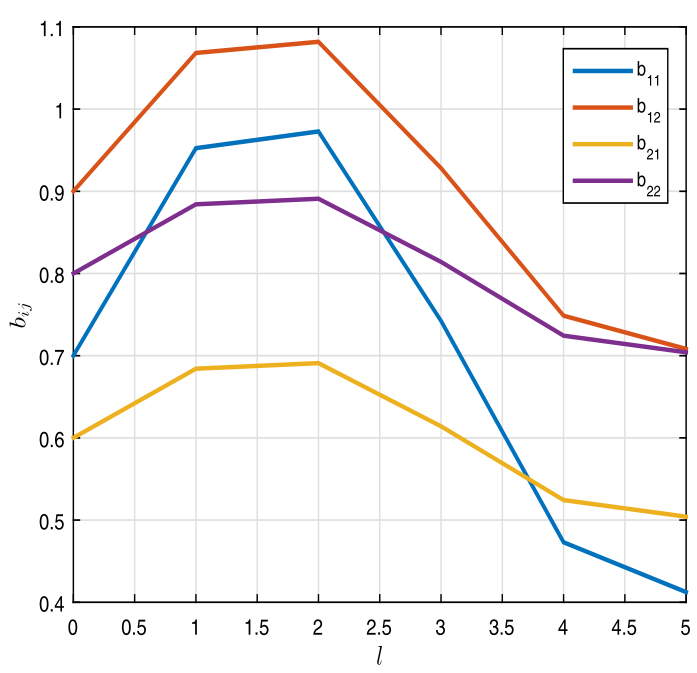

Figure 2 Graphics of $b_{i j}$, where $i, j=1,2$, in Example 1

and

$$
\mathcal{H}_{1 *}=1.2100>0.9884=\frac{a_{12}^{*}}{b_{12 *}}, \quad \mathcal{H}_{2 *}=2.9090>2.1820=\frac{a_{21}^{*}}{b_{21 *}} .
$$

By employing Algorithms 1 and 2, we can compute the above results.

Thus assumptions (1), (2), and (3) all hold for system (25) from the main Theorem 3. Thus, Theorem 3 yields that system (25) has at least one $2 \pi$-periodic solution.

\section{Conclusions and discussion}

In the last decades, Nicholson's blowflies model has found successful applications in many areas such as population dynamics, system control theory, biomathematics, and optimization problems. In this paper, we study a discrete Nicholson's dual system with density- 
Table 1 Numerical results of $a_{i j}$ and $b_{i j}$, where $i=1,2$ and $j=1,2$, in Example 1

\begin{tabular}{|c|c|c|c|c|c|c|c|c|}
\hline \multirow[t]{2}{*}{$v$} & \multicolumn{4}{|l|}{$a_{i j}$} & \multicolumn{4}{|l|}{$b_{i j}$} \\
\hline & $\overline{a_{11}}$ & $a_{12}$ & $a_{21}$ & $a_{22}$ & $\overline{b_{11}}$ & $b_{12}$ & $b_{21}$ & $b_{22}$ \\
\hline 0 & 0.9000 & 0.7000 & 1.1000 & 1.0000 & 0.7000 & 0.9000 & 0.6000 & 0.8000 \\
\hline 1 & 0.9000 & 0.7000 & 1.1000 & 1.0000 & 0.7005 & 0.9003 & 0.6002 & 0.8002 \\
\hline 2 & 0.9000 & 0.7000 & 1.1000 & 1.0000 & 0.7010 & 0.9007 & 0.6003 & 0.8003 \\
\hline 3 & 0.9000 & 0.7000 & 1.1000 & 1.0000 & 0.7016 & 0.9010 & 0.6005 & 0.8005 \\
\hline 4 & 0.9000 & 0.7000 & 1.1000 & 1.0000 & 0.7021 & 0.9014 & 0.6007 & 0.8007 \\
\hline 5 & 0.9000 & 0.7000 & 1.1000 & 1.0000 & 0.7026 & 0.9017 & 0.6009 & 0.8009 \\
\hline 6 & 0.9000 & 0.7000 & 1.1000 & 1.0000 & 0.7031 & 0.9021 & 0.6010 & 0.8010 \\
\hline 7 & 0.9000 & 0.7000 & 1.1000 & 1.0000 & 0.7037 & 0.9024 & 0.6012 & 0.8012 \\
\hline 8 & 0.9000 & 0.7000 & 1.1000 & 1.0000 & 0.7042 & 0.9028 & 0.6014 & 0.8014 \\
\hline 9 & 0.9000 & 0.7000 & 1.1000 & 1.0000 & 0.7047 & 0.9031 & 0.6016 & 0.8016 \\
\hline 10 & 0.9000 & 0.7000 & 1.1000 & 1.0000 & 0.7052 & 0.9035 & 0.6017 & 0.8017 \\
\hline 11 & 0.9000 & 0.7000 & 1.1000 & 0.9999 & 0.7058 & 0.9038 & 0.6019 & 0.8019 \\
\hline 12 & 0.9000 & 0.7000 & 1.1000 & 0.9999 & 0.7063 & 0.9042 & 0.6021 & 0.8021 \\
\hline 13 & 0.9000 & 0.7000 & 1.0999 & 0.9999 & 0.7068 & 0.9045 & 0.6023 & 0.8023 \\
\hline 14 & 0.9000 & 0.7000 & 1.0999 & 0.9999 & 0.7073 & 0.9049 & 0.6024 & 0.8024 \\
\hline 15 & 0.9000 & 0.7000 & 1.0999 & 0.9999 & 0.7079 & 0.9052 & 0.6026 & 0.8026 \\
\hline 16 & 0.9000 & 0.7000 & 1.0999 & 0.9999 & 0.7084 & 0.9056 & 0.6028 & 0.8028 \\
\hline 17 & 0.9000 & 0.7000 & 1.0999 & 0.9999 & 0.7089 & 0.9059 & 0.6030 & 0.8030 \\
\hline 18 & 0.9000 & 0.7000 & 1.0999 & 0.9999 & 0.7094 & 0.9063 & 0.6031 & 0.8031 \\
\hline 19 & 0.8999 & 0.6999 & 1.0999 & 0.9998 & 0.7099 & 0.9066 & 0.6033 & 0.8033 \\
\hline
\end{tabular}

dependent morality harvesting terms. Some sufficient conditions for the existence of positive periodic solutions have been established. Moreover, a numerical example is given to show the feasibility of our results. Also, this result relates to biological modeling $[5,16,59]$.

\section{Appendix: Supporting information}

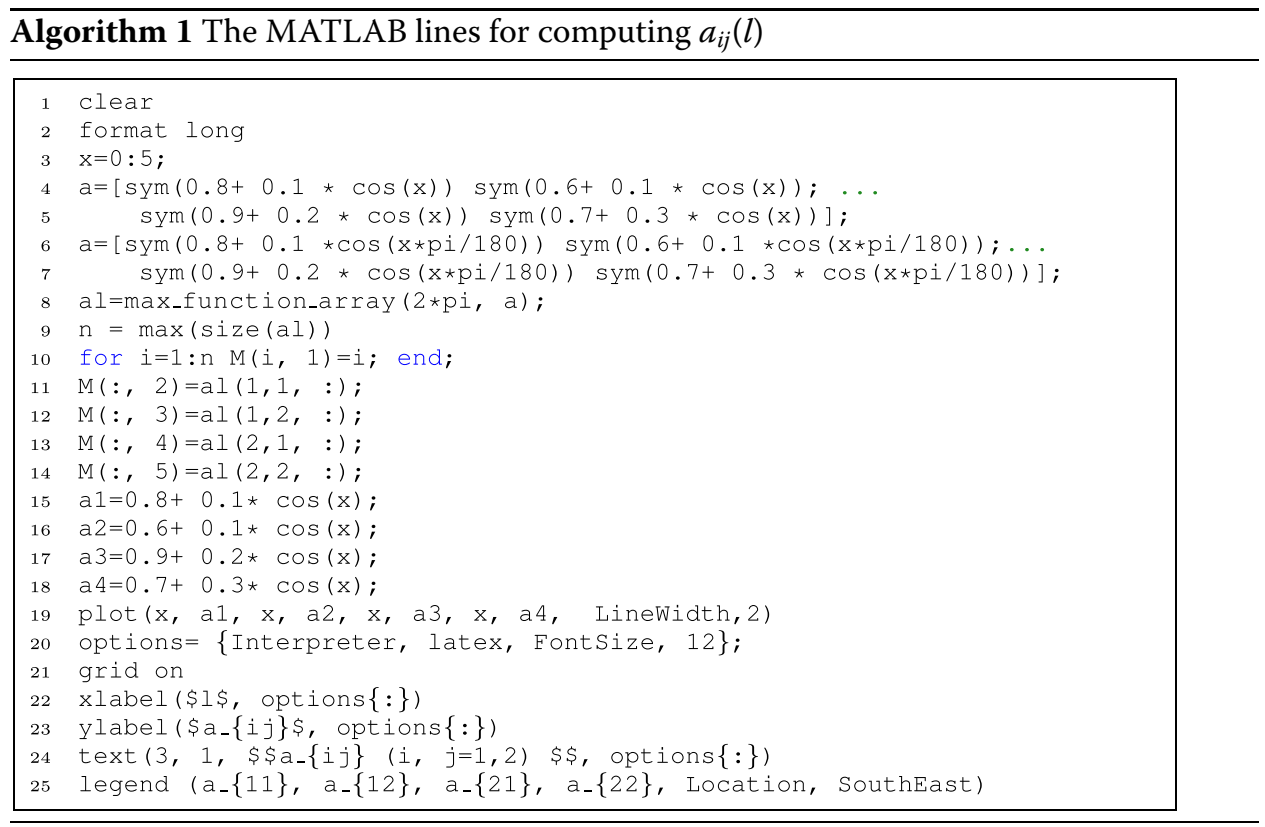




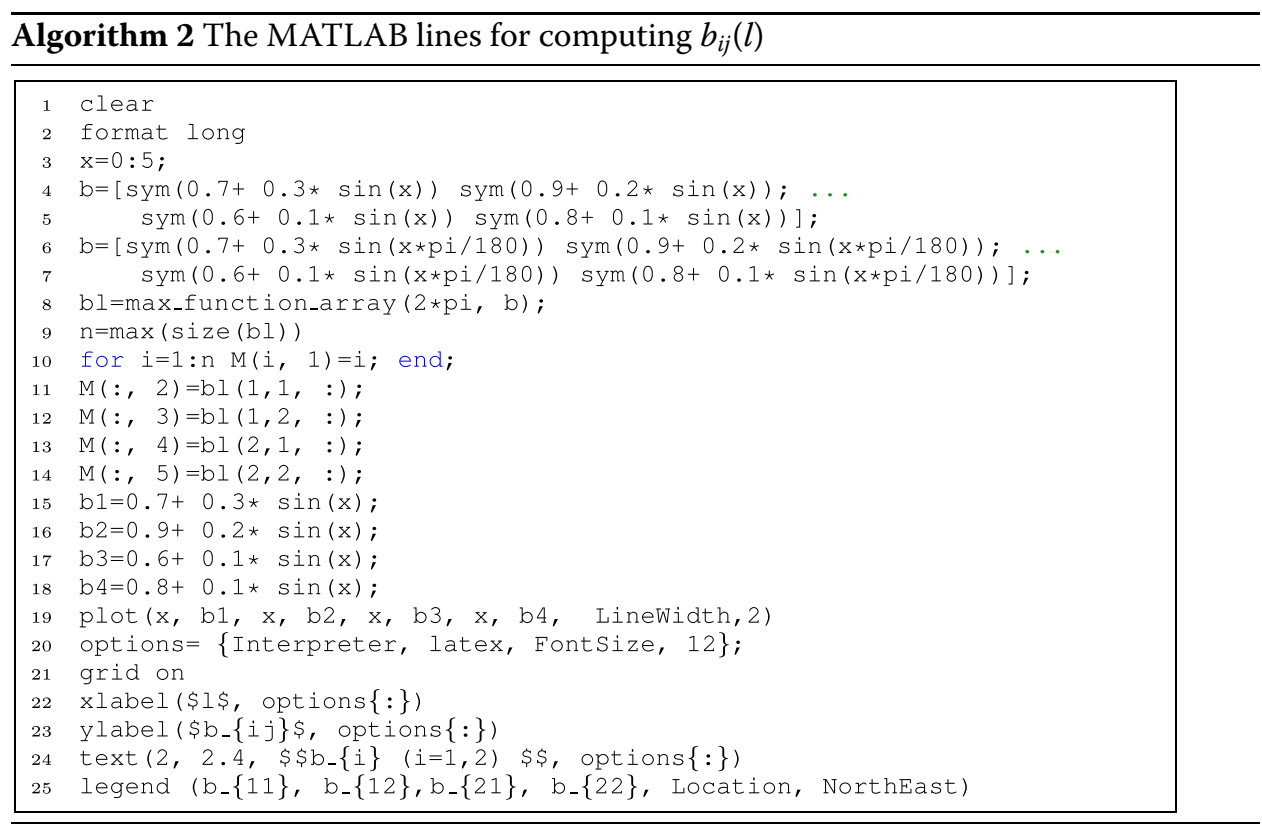

\section{Acknowledgements}

The authors would like to thank the editors and the anonymous reviewers for their constructive comments and suggestions that have helped to improve the present paper. J. Alzabut would like to thank Prince Sultan University for supporting this work. The third author was supported by Bu-Ali Sina University.

Funding

Not applicable.

Availability of data and materials

Data sharing not applicable to this article as no datasets were generated or analyzed during the current study.

Ethics approval and consent to participate

Not applicable.

\section{Competing interests}

The authors declare that they have no competing interests.

\section{Consent for publication}

Not applicable.

\section{Authors' contributions}

The authors declare that the study was realized in collaboration with equal responsibility. All authors read and approved the final manuscript.

\section{Author details}

'Department of Mathematics, Bharathidasan University, Tiruchirappalli, 620 024, Tamil Nadu, India. ${ }^{2}$ Department of Mathematics and General Sciences, Prince Sultan University, Riyadh, Saudi Arabia. ${ }^{3}$ Department of Industrial Engineering, OSTIM Technical University, 06374, Ankara, Turkey. ${ }^{4}$ Department of Mathematics, Bu-Ali Sina University, Hamedan, Iran. ${ }^{5}$ Department of Medical Research, China Medical University Hospital, China Medical University, Taichung, Taiwan.

${ }^{6}$ School of Mathematics, Hefei Normal University, Hefei, 230039, P.R. China.

\section{Publisher's Note}

Springer Nature remains neutral with regard to jurisdictional claims in published maps and institutional affiliations.

Received: 10 March 2021 Accepted: 21 July 2021 Published online: 31 July 2021

\section{References}

1. Zhou, T.: Global Stability. Springer, New York (2013). https://doi.org/10.1007/978-1-4419-9863-7-497

2. Alligood, K.T., Sauer, T.D., Yorke, J.A.: An Introduction to Dynamical Systems. Springer, New York (1996)

3. Sweilam, N., Al-Mekhlafi, S., Shatta, S., Baleanud, D.: Numerical study for two types variable-order Burgers' equations with proportional delay. Appl. Numer. Math. 156, 364-376 (2020). https://doi.org/10.1016/j.apnum.2020.05.006

4. Long, X., Gong, S.: New results on stability of Nicholson's blowflies equation with multiple pairs of time-varying delays. Appl. Math. Lett. 100, 106027 (2020). https://doi.org/10.1016/j.aml.2019.106027 
5. Freedman, H.I., Gopalsamy, K.: Global stability in time-delayed single-species dynamics. Bull. Math. Biol. 48, 485-492 (1986). https://doi.org/10.1007/BF02462319

6. Jaradat, I., Alquran, M., Momani, S., Baleanud, D.: Numerical schemes for studying biomathematics model inherited with memory-time and delay-time. Alex. Eng. J. 59(5), 2969-2974 (2020). https://doi.org/10.1016/j.aej.2020.03.038

7. Sweilam, N., Al-Mekhlafi, S.M., Mohammed, Z.N., Baleanu, D.: Optimal control for variable order fractional HIV/AIDS and malaria mathematical models with multi-time delay. Alex. Eng. J. 59(5), 3149-3162 (2020), https://doi.org/10.1016/j.aej.2020.07.021

8. Duan, L., Fang, X., Huang, C.: Global exponential convergence in a delayed almost periodic Nicholson's blowflies model with discontinuous harvesting. Math. Methods Appl. Sci. 41(5), 1954-1965 (2018). https://doi.org/10.1002/mma.4722

9. Xu, Y., Cao, Q., Guo, X.: Stability on a patch structure Nicholson's blowflies system involving distinctive delays. Appl. Math. Lett. 105, 106340 (2020). https://doi.org/10.1016/j.aml.2020.106340

10. Huang, C., Long, X., Huang, L., Fu, S.: Stability of almost periodic Nicholson's blowflies model involving patch structure and mortality terms. Can. Math. Bull. 63(2), 405-422 (2020). https://doi.org/10.4153/S0008439519000511

11. Long, X.: Novel stability criteria on a patch structure Nicholson's blowflies model with multiple pairs of time-varying delays. AIMS Math. 5(6), 7387-7401 (2020). https://doi.org/10.3934/math.2020473

12. Zhang, H., Cao, Q., Yang, H.: Asymptotically almost periodic dynamics on delayed Nicholson-type system involving patch structure. J. Inequal. Appl. 2020, 102 (2020). https://doi.org/10.1186/s13660-020-02366-0

13. Qian, C., Hu, Y.: Novel stability criteria on nonlinear density-dependent mortality Nicholson's blowflies systems in asymptotically almost periodic environments. J. Inequal. Appl. 2020, 13 (2020). https://doi.org/10.1186/s13660-019-2275-4

14. Nicholson, A.J.: An outline of the dynamics of animal populations. Aust. J. Zool. 2, 9-65 (1954)

15. Gurney, W.S.C., Blythe, S.P., Nisbet, R.M.: Periodic solutions for a non-monotone family of delayed differential equations with applications to Nicholson's systems. Nature 287, 17-21 (1980)

16. Deng, K., Wu, Y.: On the diffusive Nicholson's blowflies equation with distributed delay. Appl. Math. Lett. 50, 126-132 (2015). https://doi.org/10.1016/j.aml.2015.06.013

17. Rezapour, S., Mohammadi, H., Samei, M.E.: SEIR epidemic model for COVID-19 transmission by Caputo derivative of fractional order. Adv. Differ. Equ. 2020, 490 (2020). https://doi.org/10.1186/s13662-020-02952-y

18. Huang, C., Wang, J., Huang, L.: Asymptotically almost periodicity of delayed Nicholson-type system involving patch structure. Electron. J. Differ. Equ. 2020(61), 1 (2020)

19. Ding, H.S., Ji, M.X., N'Guérékata, G.M.: Pseudo almost periodic solutions for a Nicholson's blowflies model with mortality term. J. Nonlinear Evol. Equ. Appl. 2018(1), 1-10 (2018)

20. Berezansky, L., Braverman, E., Idels, L.: Nicholson's blowflies differential equations revisited: main results and open problems. Appl. Math. Model. 30, 1405-1417 (2010)

21. Yao, Z., Alzabut, J.: Dynamics of almost periodic Nicholson's blowflies model with nonlinear density-dependent mortality terms. Ital. J. Pure Appl. Math. 38, 218-234 (2017)

22. Liu, B.: Almost periodic solutions for a delayed Nicholson's blowflies model with a nonlinear density-dependent mortality term. Adv. Differ. Equ. 2014, 72 (2014)

23. Liu, P., Zhang, L., Liu, S., Zheng, L.: Global exponential stability of almost periodic solutions for Nicholson's blowflies system with nonlinear density-dependent mortality terms and patch structure. Math. Model. Anal. 22, 484-502 (2017)

24. Chen, W., Wang, L.: Positive periodic solutions of Nicholson-type delay systems with nonlinear density-dependent mortality terms. Abstr. Appl. Anal. 2012, Article ID 843178 (2012)

25. Alzabut, J.O.: Almost periodic solutions for an impulsive delay Nicholson's blowflies model. J. Comput. Appl. Math. 234, 233-239 (2010)

26. Xia, Z., Li, Z., Chai, J.: Pseudo almost periodic dynamics of impulsive Nicholson's blowflies model with nonlinear density-dependent mortality term. Jpn. J. Ind. Appl. Math. 35, 311-333 (2018)

27. Dai, B., Bao, L.: Positive periodic solutions generated by impulses for the delay Nicholson's blowflies model. Electron. J. Qual. Theory Differ. Equ. 2016, 4 (2016)

28. Hedayati, V., Samei, M.E.: Positive solutions of fractional differential equation with two pieces in chain interval and simultaneous Dirichlet boundary conditions. Bound. Value Probl. 2019, 141 (2019)

29. Kalvandi, V., Samei, M.E.: New stability results for a sum-type fractional $q$-integro-differential equation. J. Adv. Math. Stud. 12(2), 201-209 (2019)

30. Samei, M.E., Hedayati, V., Rezapour, S.: Existence results for a fraction hybrid differential inclusion with Caputo-Hadamard type fractional derivative. Adv. Differ. Equ. 2019, 163 (2019)

31. Tan, Y.: Dynamics analysis of Mackey-Glass model with two variable delays. Math. Biosci. Eng. 17(5), 4513-4526 (2020). https://doi.org/10.3934/mbe.2020249

32. Manickam, I., Ramachandran, R., Rajchakit, G., Cao, J., Huang, C.: Novel Lagrange sense exponential stability criteria for time-delayed stochastic Cohen-Grossberg neural networks with Markovian jump parameters: a graph-theoretic approach. Nonlinear Anal., Model. Control 25(5), 726-744 (2020). https://doi.org/10.15388/namc.2020.25.16775

33. Liu, Y., Wu, J., Wang, X.: Collective periodic motions in a multiparticle model involving processing delay. Math. Methods Appl. Sci. 44(5), 3280-3302 (2021). https://doi.org/10.1002/mma.6939

34. Zhang, X., Hu, H.: Convergence in a system of critical neutral functional differential equations. Appl. Math. Lett. 2020 107 (2020). https://doi.org/10.1016/j.aml.2020.106385

35. Huang, C., Yang, L., Cao, J.: Asymptotic behavior for a class of population dynamics. AlMS Math. 5(4), 3378-3390 (2020). https://doi.org/10.3934/math.2020218

36. Huang, C., Long, $X$. ., Cao, J.: Stability of antiperiodic recurrent neural networks with multiproportional delays. Math Methods Appl. Sci. 43(9), 6093-6102 (2020). https://doi.org/10.1002/mma.6350

37. Zhang, H., Qian, C.: Convergence analysis on inertial proportional delayed neural networks. Adv. Differ. Equ. 2020, 277 (2020). https://doi.org/10.1186/s13662-020-02737-3

38. Alzabut, J., Bolat, Y., Abdeljawad, T.: Almost periodic dynamics of a discrete Nicholson's blowflies model involving a linear harvesting term. Adv. Differ. Equ. 2012, 158 (2012) 
39. Chen, X., Shi, C., Wang, Y.: Almost periodic solution of a discrete Nicholson's blowflies model with delay and feedback control. Adv. Differ. Equ. 2016, 185 (2016)

40. Saker, S.H.: Oscillation of continuous and discrete diffusive delay Nicholson's blowflies models. Appl. Math. Comput. 167, 179-197 (2005)

41. Yao, Z:: Existence and exponential convergence of almost periodic positive solution for Nicholson's blowflies discrete model with linear harvesting term. Math. Methods Appl. Sci. 37, 2354-2362 (2014)

42. Alzabut, J., Obaidat, S., Yao, Z.: Exponential extinction of discrete Nicholson's blowflies systems with patch structure and mortality terms. J. Math. Comput. Sci. 16, 298-307 (2016)

43. Li, Y., Li, B.: Existence and exponential stability of positive almost periodic solution for Nicholson's blowflies models on time scales. SpringerPlus 5, 1-24 (2016)

44. Braverman, E., Kinzebulatov, D.: Nicholson's blowflies equation with a distributed delay. Can. Appl. Math. Q. 2 , 107-128 (2006)

45. Amster, P., Déboli, A.: Necessary and sufficient conditions for the existence of periodic solutions of a Nicholson type delay system. Differ. Equ. Dyn. Syst. 2016, 1-9 (2016)

46. Wang, W., Wang, L., Chen, W.: Existence and exponential stability of positive almost periodic solution for Nicholson-type delay systems. Nonlinear Anal., Real World Appl. 12, 1938-1949 (2011)

47. Hou, X., Duan, L.: New results on periodic solutions of delayed Nicholson's blowflies models. Electron. J. Qual. Theory Differ. Equ. 2012, 24 (2012)

48. Zhao, W., Zhu, C., Zhu, H.: On positive periodic solution for the delay Nicholson's blowflies model with a harvesting term. Appl. Math. Model. 36, 3335-3340 (2012)

49. Amster, P., Déboli, A.: Existence of positive t-periodic solutions of a generalized Nicholson's blowflies model with a nonlinear harvesting term. Appl. Math. Lett. 25, 1203-1207 (2012)

50. Alzabut, J.O.: Existence and exponential convergence of almost periodic solutions for a discrete Nicholson's blowflies model with nonlinear harvesting term. Math. Sci. Lett. 2, 201-207 (2013)

51. Liu, Q.L., Ding, H.S.: Existence of positive almost periodic solutions for a Nicholson's blowflies model. Electron. J. Differ. Equ. 56, 1 (2013)

52. Xiong, W.: New results on positive pseudo-almost periodic solutions for a delayed Nicholson's blowflies model. Nonlinear Dyn. 85, 563-571 (2016)

53. Wang, W. Liu, F., Chen, W.: Exponential stability of pseudo almost periodic delayed Nicholson-type system with patch structure. Math. Methods Appl. Sci. 42, 592-604 (2019)

54. Tang, Y., Xie, S.: Global attractivity of asymptotically almost periodic Nicholson's blowflies models with a nonlinear density-dependent mortality term. Int. J. Biomath. 6, 1-15 (2018)

55. Long, F., Yang, M.: Positive periodic solutions of delayed Nicholson's blowflies model with a linear harvesting term Electron. J. Qual. Theory Differ. Equ. 2011, 41 (2011)

56. Faria, T: Periodic solutions for a non-monotone family of delayed differential equations with applications to Nicholson's systems. J. Differ. Equ. 2017, 1-25 (2017)

57. Gaines, R., Mawhin, J.: Coincidence Degree and Nonlinear Differential Equations. Berlin (1977)

58. Zhang, R.Y., Wang, Z.C., Chen, Y., Wu, J.: Periodic solutions of a single species discrete population model with periodic harvest/stock. Comput. Math. Appl. 39, 79-90 (2000)

59. Readshaw, J.L., Cuff, W.R.: A model of Nicholson's blowfly cycles and its relevance to predation theory. J. Anim. Ecol. 49(3), 1005-1010 (1986). https://doi.org/10.2307/4241

\section{Submit your manuscript to a SpringerOpen ${ }^{\circ}$ journal and benefit from:}

- Convenient online submission

- Rigorous peer review

- Open access: articles freely available online

- High visibility within the field

- Retaining the copyright to your article

Submit your next manuscript at $\gg$ springeropen.com 